\title{
Rapid effect of nicotine intake on neuroplasticity in non-smoking humans
}

\section{Jessica Grundey ${ }^{1}$, Nivethida Thirugnanasambandam ${ }^{1}$, Kim Kaminsky $^{1}$, Anne Drees ${ }^{1}$, Angela C. Skwirba ${ }^{1}$, Nicolas Lang ${ }^{2}$, Walter Paulus ${ }^{1}$ and Michael A. Nitsche ${ }^{1}$}

${ }^{1}$ Department of Clinical Neurophysiology, Georg-August-University, Göttingen, Germany

${ }^{2}$ Department of Neurology, Christian-Albrechts University, Kiel, Germany

\section{Edited by:}

Valentina Echeverria Moran, Bay Pines

VA Medical Center, USA

Reviewed by:

Erik Wong, AstraZeneca, USA

Jason B. Wu, Cedars-Sinai Medical

Center, USA

Valentina Echeverria Moran, Bay Pines

VA Medical Center, USA

*Correspondence:

Jessica Grundey, Department of

Clinical Neurophysiology,

Georg-August-University, Robert-Koch

Street 40, Göttingen 37075, Germany.

e-mail: jgrundey@med.

uni-goettingen.de
In various studies nicotine has shown to alter cognitive functions in non-smoking subjects. The physiological basis for these effects might be nicotine-generated modulation of cortical structure, excitability, and activity, as mainly described in animal experiments. In accordance, a recently conducted study demonstrated that application of nicotine for hours via nicotine patch in non-smoking humans alters the effects of neuroplasticity-inducing non-invasive brain stimulation techniques on cortical excitability. Specifically, nicotine abolished inhibitory plasticity independent from the focality of the stimulation protocol. While nicotine prevented also the establishment of non-focal facilitatory plasticity, focal synapsespecific facilitatory plasticity was enhanced. These results agree with a focusing effect of prolonged nicotine application on facilitatory plasticity. However, since nicotine induces rapid adaption processes of its receptors, this scenario might differ from the effect of nicotine in cigarette smoking. Thus in this study we aimed to gain further insight in the mechanism of nicotine on plasticity by exploring the effect of nicotine spray on non-focal and focal plasticity-inducing protocols in non-smoking subjects, a fast-acting agent better comparable to cigarette smoking. Focal, synapse-specific plasticity was induced by paired associative stimulation (PAS), while non-focal plasticity was elicited by transcranial direct current stimulation (tDCS). Forty eight non-smokers received nicotine spray respectively placebo combined with one of the following protocols (anodal tDCS, cathodal tDCS, PAS-25, and PAS-10). Corticospinal excitability was monitored via motor-evoked potentials elicited by transcranial magnetic stimulation (TMS). Nicotine spray abolished facilitatory plasticity irrespective of focality and PAS-10-induced excitability diminution, while tDCS-derived excitability reduction was delayed and weakened. Nicotine spray had thus a clear effect on neuroplasticity in non-smoking subjects. However, the effects of nicotine spray differ clearly from those of prolonged nicotine application, which might be due to missing adaptive nicotinic receptor alterations. These results enhance our knowledge about the dynamic impact of nicotine on plasticity, which might be related to its heterogenous effect on cognition.

Keywords: neuroplasticity, nicotine, non-smokers, PAS, tDCS

\section{INTRODUCTION}

Nicotine binds to the nicotinergic type of cholinergic receptors, which are ligand-gated cation channels. Nicotine also affects other transmitter systems by regulating the release of dopamine, adrenaline, serotonin, and glutamate, amongst others. Hereby nicotine is thought to be critically involved in the induction and modulation of neuroplasticity (Burnashev, 1998; Dajas-Bailador and Wonnacott, 2004; Levin et al., 2006), the likely physiological basis of learning and memory formation (Rioult-Pedotti et al., 1998, 2000). Specifically, in animal experiments the activation of nicotinic receptors results in a facilitation of long-term potentation (LTP), both dependent and independent of NMDA-receptor activation (Sawada et al., 1994; Huerta and Lisman, 1995; Matsuyama et al., 2000).

\section{COGNITIVE EFFECTS OF NICOTINE}

On a functional basis, in animal experiments nicotine has been shown to improve working memory function (Levin et al., 1994) and attention (Hahn and Stolerman, 2002), while in humans cognitive results are heterogeneous. Kleykamp et al. (2005) have found no effect of nicotine gum in different doses on attention and working memory in never-smokers. In contrast, other studies have shown that nicotine improves alerting attention-accuracy (Barr et al., 2008), visuospatial attention (Thiel et al., 2005), and working memory (Kumari et al., 2003) in non-smoking subjects. However, nicotinic plasticity modulation has been explored only in few studies in humans so far.

\section{NICOTINIC PLASTICITY MODULATION}

Recently it was shown that global cholinergic activation via the cholinesterase-inhibitor rivastigmine enhances focal plasticity induced by paired associative stimulation (PAS) and abolishes/reverses non-focal facilitatory plasticity generated by transcranial direct current stimulation (tDCS), thus resulting in a focusing effect of acetylcholine on facilitatory plasticity (Kuo et al., 2007). Similar results have been found for nicotinic effects 
in non-smokers (Thirugnanasambandam et al., 2011). However, in difference to global cholinergic activation, nicotine reduced, or abolished inhibitory plasticity in these subjects. In principal accordance, nicotine enhanced the facilitatory effects of intermittent theta burst stimulation in the human motor cortex (Swayne et al., 2009). However, nicotine pharmacokinetics in the abovementioned study of Thirugnanasambandam et al. (2011) differ from that present in cigarette smokers, because in that study nicotine patches were applied, which are characterized by a slow build-up and long duration of enhanced nicotine concentration, whereas cigarette smoking results in a fast build-up and decay of nicotine concentration. Since nicotinic receptor activation induces rapid adaptive processes like desensitization (Alkondon et al., 2000; Mansvelder and McGehee, 2000), upregulation, and greater density of AChR (Flores et al., 1992; Mukhin et al., 2008) these might have affected the impact of nicotine on plasticity relevantly in that study.

\section{PLASTICITY INDUCTION PROTOCOLS}

In the present study, we therefore aimed to mimic the pharmacokinetics of cigarette smoking by exploring the impact of nicotine spray, which results in maximal nicotine plasma concentration within a few minutes, on focal and non-focal plasticity. Facilitatory and inhibitory plasticity were induced by tDCS (Nitsche and Paulus, 2000, 2001) and PAS (Stefan et al., 2000). Both stimulation protocols induce non-invasively NMDA- and calcium channeldependent plasticity (Stefan et al., 2002; Nitsche et al., 2003, 2004), though tDCS is supposed to induce non-focal plasticity changes due to affecting large neuron populations under large electrodes (Purpura and McMurtry, 1965; Nitsche et al., 2007), while PASinduced plasticity is restricted to synaptic connections between somatosensory and motor cortex (Weise et al., 2006). As outlined above, in non-smokers nicotine patch abolished inhibitory plasticity and focused facilitatory plasticity. Since nicotine receptors are rapidly modified by chronic nicotine exposure, we hypothesize that administration of a single dose of fast-acting nicotine might affect plasticity differently as compared to nicotine patch.

\section{MATERIALS AND METHODS SUBJECTS}

Altogether 48 otherwise healthy non-smoking subjects participated in this study. Table 1 displays the characteristics of the subjects in terms of age and gender. All subjects were of Caucasian origin. Chronic and acute medical diseases or any history of neurological/psychiatric disease were excluded before entering the study by assessment of medical history, likewise intake of chronic and acute medication. Pregnancy, family history of epilepsy, presence of any metallic implant, or cardiac pacemaker were ruled out. All subjects gave written informed consent before participating in the study. The experiments were approved by the local Ethics Committee and conformed to the principles laid down in the Declaration of Helsinki. Allocation of the subjects to the respective experimental conditions as well as order of sessions was randomized.

\section{PAIRED ASSOCIATIVE STIMULATION}

Altogether twenty-four subjects participated in the PAS experiment. Twelve non-smokers participated in the inhibitory PAS
Table 1 | Characteristics of the subjects participating in the experiments.

\begin{tabular}{lllll}
\hline Stimulation Parameter & $\begin{array}{l}\text { Anodal } \\
\text { tDCS }\end{array}$ & $\begin{array}{l}\text { Cathodal } \\
\text { tDCS }\end{array}$ & PAS-10 & PAS-25 \\
\hline Number of subjects & 12 & 12 & 12 & 12 \\
Number of females(\%) & $6(50)$ & $7(58)$ & $6(50)$ & $6(50)$ \\
Age & $24.4 \pm$ & $26.9 \pm$ & $25.9 \pm$ & $24.5 \pm$ \\
& 1.2 & 3.6 & 2.1 & 1.3 \\
S1 mV before nicotine spray & 41.3 & 45.6 & 43.9 & 43.6 \\
S1 mV after nicotine spray & 42.3 & 44.9 & 44.4 & 43.3 \\
\hline
\end{tabular}

protocol (PAS-10) and 12 in the excitatory PAS protocol (PAS25). Peripheral nerve stimulation was delivered to the right ulnar nerve at the wrist level by a Digitimer D185 multipulse stimulator (Digitimer, Welwyn Garden City, UK) at an intensity of $300 \%$ of the sensory perceptual threshold followed by single pulse transcranial magnetic stimulation (TMS) applied with a stimulator output resulting in motor-evoked potentials (MEPs) of approximately $1 \mathrm{mV}$ amplitude ("baseline intensity," see description in Section "Monitoring of Cortical Excitability"). The paired pulses were repeated 90 times at a frequency of $0.05 \mathrm{~Hz}$. This protocol induces long-lasting excitability changes in the motor cortex depending on the interstimulus interval (ISI). An ISI of $10 \mathrm{~ms}$ induces excitability diminution (PAS-10) whereas an ISI of $25 \mathrm{~ms}$ induces facilitation (PAS-25; Stefan et al., 2000; Wolters et al., 2003). The PAS-protocols were combined with either nicotine or placebo spray for each subject in different experimental sessions.

\section{TRANSCRANIAL DIRECT CURRENT STIMULATION}

Twenty four subjects participated in the tDCS experiments (12 non-smoking subjects participated in the inhibitory tDCS protocol and 12 in the excitatory tDCS protocol). We used a batterydriven constant current stimulator (Schneider Electronics, Gleichen, Germany) with a maximum output of $2 \mathrm{~mA}$. tDCS was administered via rubber electrodes covered by saline soaked sponges $\left(35 \mathrm{~cm}^{2}\right)$. One electrode was positioned over the motor cortex representational area of the right abductor digiti minimi muscle (ADM), the other electrode above the right orbit. All subjects received $1 \mathrm{~mA}$ of either anodal or cathodal stimulation for 13 min (anodal tDCS) or 9 min (cathodal tDCS), which had been demonstrated to induce cortical excitability enhancement or inhibition lasting for about $1 \mathrm{~h}$ after the end of stimulation (Nitsche and Paulus, 2001; Nitsche et al., 2003) combined with nicotine spray or placebo medication in different experimental sessions.

\section{Monitoring of motor cortex excitability}

Transcranial magnetic stimulation-elicited MEPs were recorded to measure excitability changes of the representational motor cortical area of the right ADM. Single pulse TMS was conducted by a Magstim 200 magnetic stimulator (Magstim Company, Whitland, Dyfed, UK) at a frequency of $0.25 \mathrm{~Hz}$ with a figure of eight-shaped coil (diameter of one winding $70 \mathrm{~mm}$, peak magnetic field, 2.2 $\mathrm{T})$. The coil was held tangentially to the scalp at an angle of $45^{\circ}$ to the sagittal plane with the coil handle pointing laterally and posterior. The optimal position was defined as the site where 
stimulation resulted consistently in the largest MEPs. Surface EMG was recorded from the right $\mathrm{ADM}$ with $\mathrm{Ag}-\mathrm{AgCl}$ electrodes in a belly tendon montage. The signals were amplified and filtered with a time constant of $10 \mathrm{~ms}$ and a low-pass filter of $2.5 \mathrm{kHz}$, then digitized at an analog-to-digital rate of $5 \mathrm{kHz}$ and further relayed into a laboratory computer using the Signal software and CED 1401 hardware (Cambridge Electronic Design). The intensity was adjusted to elicit, on average, baseline MEPs of $1 \mathrm{mV}$ peak-to-peak amplitude, and was kept constant for the post-stimulation.

\section{PHARMACOLOGICAL INTERVENTION}

Each subject participated in two sessions in randomized order. Nasal spray contained nicotine or inactive placebo. Nicotine was administered by nasal spray, containing $10 \mathrm{mg} / \mathrm{ml}$ nicotine, in a cumulative dose of $1 \mathrm{mg}$ (Nicorette ${ }^{\circledR}$ Nasal Spray, McNeil Products, UK) to all subjects in combination with one of the stimulation protocols, anodal tDCS, cathodal tDCS, PAS-10, and PAS-25. The rise time of nicotine by nasal spray in venous blood levels is close to venous blood levels of nicotine delivered by cigarets (Schneider et al., 1995) with a plasma peak level after 5-10 min. Side effects of the nasal nicotinic administration were coughing, sneezing, throat irritation, and dizziness like it has been described from prior clinical trials (Sutherland et al., 1992). Symptoms subsided rapidly after some minutes. To have a comparability to the nicotine patch study, we chose a dose of nicotine spray $(1 \mathrm{mg})$ that delivers nicotine blood levels comparable to those of nicotine patch $(8-9 \mathrm{ng} / \mathrm{ml}$, see also Tønnesen et al., 1991; Pomerleau et al., 1992).

\section{COURSE OF THE EXPERIMENT}

Subjects were seated comfortably in a reclined chair with headand armrest and asked to relax completely. EMG electrodes were placed at the right ADM as described above. Their exact position was marked with a pen. Then TMS was applied over the left representational area of the right $\mathrm{ADM}$ to determine the spot with the consistently highest MEPs in the resting ADM (optimal site), which was then marked with a pen. The TMS-intensity was adjusted to elicit MEP amplitudes of $1 \mathrm{mV}(\mathrm{S} 1 \mathrm{mV})$. Twenty MEPs were recorded at this stimulus intensity and the mean MEP amplitude was calculated at baseline. Then nicotine nasal spray respective placebo spray was administered. Side effects like coughing and sneezing, that could interfere with the measurements subsided quickly and $10 \mathrm{~min}$ later one of the stimulation protocols, either tDCS or PAS, was administered, followed by immediate recording of at least $20 \mathrm{MEPs}$ at the time points of $0,5,10,15,20$, $25,30,60,90$, and $120 \mathrm{~min}$. For the nicotine spray condition, the after-measurements were also conducted the evening of the stimulation day and in the morning and evening of the day following the plasticity induction procedure. Sessions were conducted in randomized order, and an inter-session interval of at least 1 week was obligatory to avoid interferences. See also Figure 1 for course of the experiment.

\section{DATA ANALYSIS AND STATISTICS}

For all subjects the means of $20 \mathrm{MEP}$ amplitudes recorded at each time point was calculated. The post-intervention mean MEP amplitudes from each subject were then normalized to the respective individual mean baseline MEP amplitude (quotient of postvs. pre-intervention MEP amplitudes). Statistical analysis used SPSS general linear model analysis for variances for repeated measurements on normalized data. MEP amplitude was the dependent variable including all time points up to $120 \mathrm{~min}$ after stimulation. Drug (nicotine vs. plc) and time points were included as within-subjects factors. Stimulation protocol (atDCS, ctDCS,

\section{Medication}

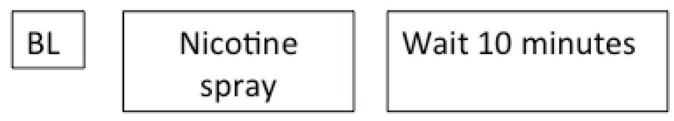

TMS

\section{Plasticity induction \\ TMS monitoring of cortex excitability}

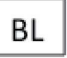
atDCS; ctDCS; PAS-25; PAS10

MEPs until the next morning after intervention

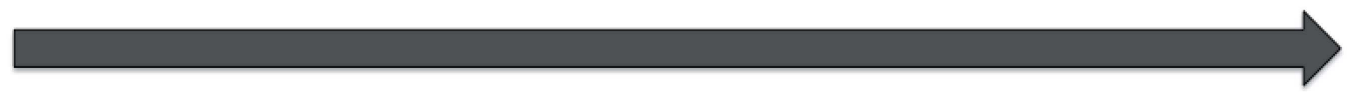

Time course

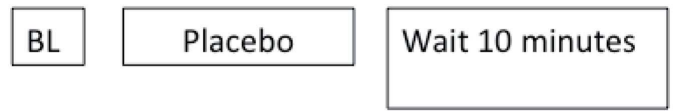

FIGURE 1 |This figure illustrates the experimental protocol of our study. At the beginning of the session baseline measurements $(B L)$ were performed and followed by administration of either nicotine spray or placebo spray. After 10 min baseline (BL) motor cortical excitability was redetermined via TMS-induced motor-evoked potentials (MEP). One of the four

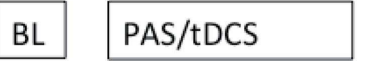

MEP until 120 minutes after intervention excitability-inducing protocols were then applied (atDCS, ctDCS, PAS-10, PAS-25). After-measurements started immediately after the application of the protocols, and were conducted every $5 \mathrm{~min}$ for the first $30 \mathrm{~min}$, then every $30 \mathrm{~min}$ up to $120 \mathrm{~min}$ for both sessions. MEP amplitudes were also recorded the next morning and next evening for the nicotine spray sessions. 
PAS-25, and PAS-10) served as between-subject factors. Mauchly's sphericity test was performed and Greenhouse-Geisser correction was applied when necessary. Student's $t$-tests (paired samples, two-tailed, $p<0.05$, not adjusted for multiple comparisons) were performed to compare the MEP amplitudes before and after the interventional brain stimulations in each condition and between drug conditions (nicotine/placebo) for each time point. A $p$-value of $<0.05$ was considered significant for all statistical analyses. Significances of differences in demographic factors were tested by one-way ANOVA and chi-square for gender. All data are expressed as mean \pm standard of error (SEM).

\section{RESULTS}

All subjects tolerated the experiments well, even though nearly all of the subjects (44 of 46 subjects, 96\%) complaint about sneezing and coughing after inhalation of nicotine spray. No significant group differences were found in terms of age, gender, TMS-intensity to elicit an MEP of $1 \mathrm{mV}(\mathrm{S} 1 \mathrm{mV})$ before and after administration of nicotine spray (see Table 1). Absolute baseline MEP amplitudes did not differ significantly within and between stimulation groups and medication conditions (Student's $t$-test, two-tailed, unpaired/paired, $p>0.05$ for all cases). The ANOVA revealed a significant main effect of the between-subject factor timepoint and stimulation. The interactions drug $\times$ stimulation, timepoint $\times$ stimulation, and drug $\times$ timepoint $\times$ stimulation were also significant (see also Table 2 for results of degrees of freedom, $F$-value, $p$-value, *indicates significant values with $p<0.05$ ).

\section{EFFECTS OF NICOTINE SPRAY ON tDCS-INDUCED PLASTICITY IN NON-SMOKERS}

In the PLC condition the anodal tDCS-induced excitability increased MEP amplitudes stayed significant until 90 min after stimulation, and the cathodal tDCS-induced inhibition lasted until $90 \mathrm{~min}$ after tDCS. As revealed by the post hoc $t$-test (paired, two-tailed, $p<0.05$ ) nicotine spray abolished the atDCS-induced long-lasting excitability enhancements in non-smoking subjects. For the cathodal tDCS protocol under influence of nicotine spray excitability diminuation started delayed after $15 \mathrm{~min}$ and lasted only until $20 \mathrm{~min}$ after stimulation. A second diminuation peak could be seen after $90 \mathrm{~min}$ and lasted until $120 \mathrm{~min}$ post-stimulation (Figure 2). Thus nicotine spray administration delayed and weakened the ctDCS-induced after-effects.

Table 2 | Results of the ANOVA.

\begin{tabular}{llll}
\hline Parameters & $\boldsymbol{d f}$ & $\boldsymbol{F}$-Value & $\boldsymbol{p}$-value \\
\hline Drug & 1 & 2.288 & 0.138 \\
Timepoint & 6.932 & 2.163 & $0.038^{*}$ \\
Stimulation & 3 & 11.423 & $0.001^{*}$ \\
Drug $\times$ stimulation & 3 & 8.673 & $0.001^{*}$ \\
Timepoint $\times$ stimulation & 30 & 2.056 & $0.001^{*}$ \\
Drug $\times$ timepoint & 5.446 & 1.478 & $0.192^{*}$ \\
Drug $\times$ timepoint $\times$ stimulation & 30 & 1.831 & $0.027^{*}$ \\
\hline
\end{tabular}

${ }^{*} p<0.05$

\section{EFFECTS OF NICOTINE SPRAY ON PAS-INDUCED PLASTICITY IN NON-SMOKERS}

As revealed by Student's $t$-test (paired, two-tailed, $p<0.05$ ) in the PLC- condition PAS-25-induced excitability changes (MEPenhancements) were significantly increased for up to $90 \mathrm{~min}$ after stimulation, while the PAS-10-induced long-lasting after-effects returned to baseline $120 \mathrm{~min}$ after PAS. Nicotine spray abolished both the PAS-10-induced inhibitory MEP-changes and the PAS25-induced excitatory after-effects completely (Figure 3). Thus nicotine spray prevented the induction of PAS-induced focal plasticity.

\section{EFFECT OF NICOTINE SPRAY ON MOTOR CORTEX EXCITABILITY MEASURED BY TMS-ELICITED MOTOR-EVOKED POTENTIALS}

To rule out, that nicotine spray itself increases or decreases motor cortex excitability measured by TMS, we compared TMS-intensity needed to elicit an MEP of $1 \mathrm{mV}$ before and after the administration of nicotine spray. The respective Student's $t$-test (paired, twotailed) did not reveal any significant difference between different stimulation groups and before vs. after nicotine (see Figure 4).

\section{DISCUSSION}

The results of the present study show that nicotine administration has a prominent and rapidly evolving effect on stimulationinduced neuroplasticity in the human primary motor cortex. In non-smoking humans, nicotine spray prevented completely the induction of focal and non-focal plasticity, as induced by PAS-25, and anodal tDCS. Moreover it reduced excitability-diminishing plasticity accomplished by PAS-10, whereas nicotine had no major impact on cathodal tDCS-generated plasticity.

These results are only in partial accordance with those of a former study in our group, where we explored the effects of longacting nicotine, administered via patch, on plasticity induced by the same stimulation techniques (Thirugnanasambandam et al., 2011). Here nicotine abolished inhibitory plasticity regardless of its focality, whereas facilitatory plasticity was enhanced, when the induction procedure was focal, but abolished, when it was non-focal. Moreover, the abolishing effect of nicotine spray on facilitatory plasticity in the present study differs prominently from the effects of nicotine lozenge application, which enhanced facilitatory plasticity induced by intermittent theta burst stimulation of the human motor cortex (Swayne et al., 2009). The comparison between the present study and that of Thirugnanasambandam, in which resulting nicotine plasma level should be more or less identical, is in favor for an impact of the duration of nicotine application on human cortical plasticity. One reason for these different effects of acute (in terms of minutes), and chronic (in terms of hours) nicotine administration on plasticity might be adaptive receptor up- or downregulation, which takes place rapidly in nicotinic receptors (Flores et al., 1992; Alkondon et al., 2000; Mukhin et al., 2008; Mansvelder and McGehee 2000) and thus might have had an impact on the results of the patch study. With regard to the study of Swayne and co-workers, some other aspects differ between the respective studies, such as kind of stimulation protocol, duration of plasticity induced by the stimulation protocol alone, expected plasma level, and pharmacokinetics, which makes a comparison between studies difficult, but nevertheless is 

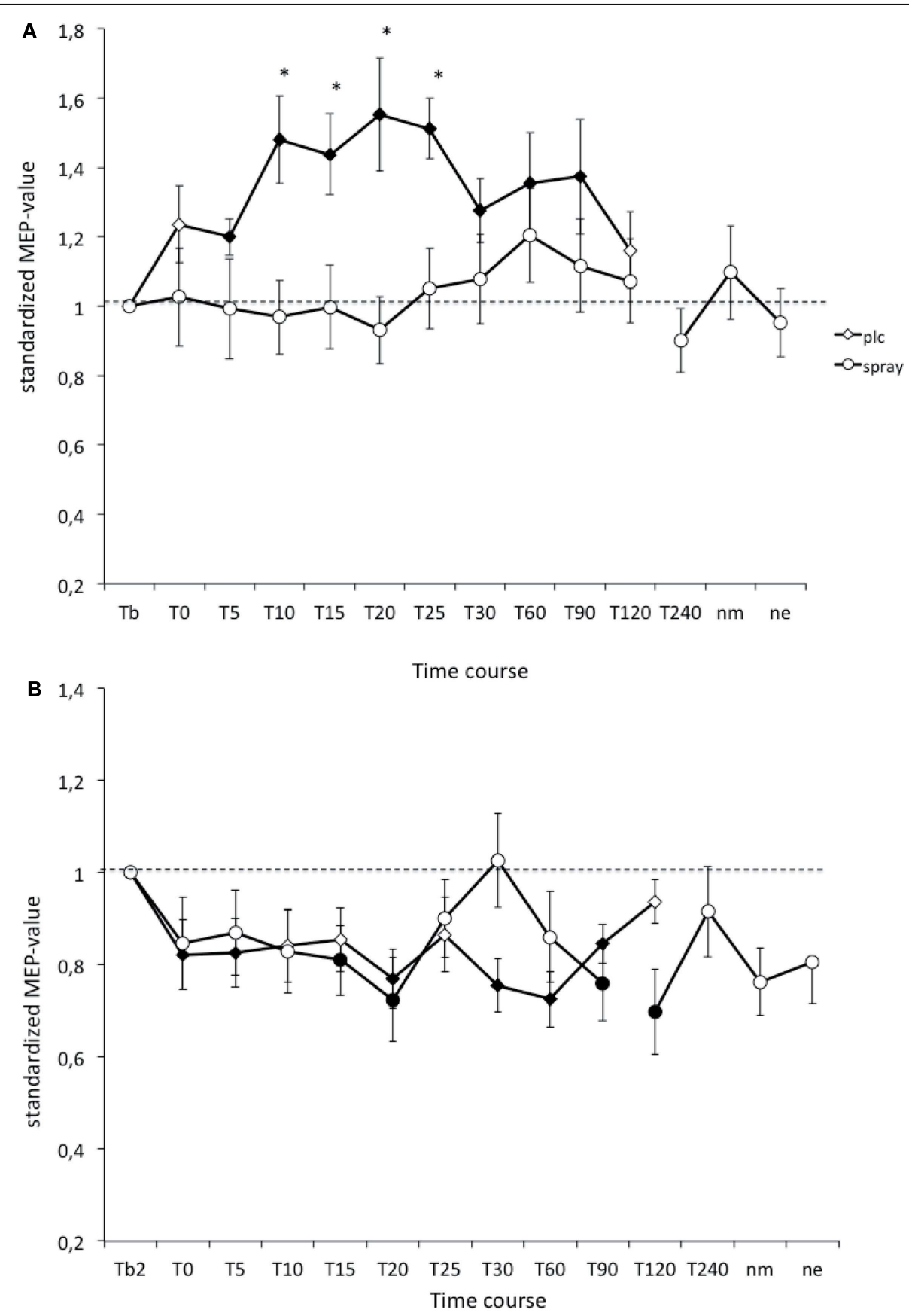

FIGURE 2 | (A,B) Nicotinergic impact on transcranial direct current stimulation (tDCS) induced neuroplasticity. Shown are the graphs with motor evoked potentials (MEP) standardized to the baseline on the $Y$-axis plotted against different time points post-intervention on the $X$-axis. Filled symbols indicate statistically significant deviations from baseline and asterisks indicate significant differences between the control and nicotine conditions (Student's $t$-test, paired, two-tailed, $p<0.05$ ). nm, Next morning; ne, next evening; plc, placebo. Tb, Baseline MEP-amplitude before begin of the stimulation protocols (standardized). Error bars indicate standard error of mean. in advance for a neuromodulatory effect of nicotine on plasticity, whose direction might be determined by diverse factors.

\section{PROPOSED MECHANISM OF ACTION}

The results of the present study allow no definite conclusions about how nicotine has affected plasticity in the present study, but some candidate mechanisms can be derived from the more general functions of nicotinic receptors, and the physiological basis of tDCS, and PAS. Nicotine binds to nicotinergic ACh-receptors that are widely distributed throughout the brain (Albuquerque et al., 2009). In the brain two major subunits compositions exist, the heteromeric assembly of $\alpha 4 \beta 2$ 

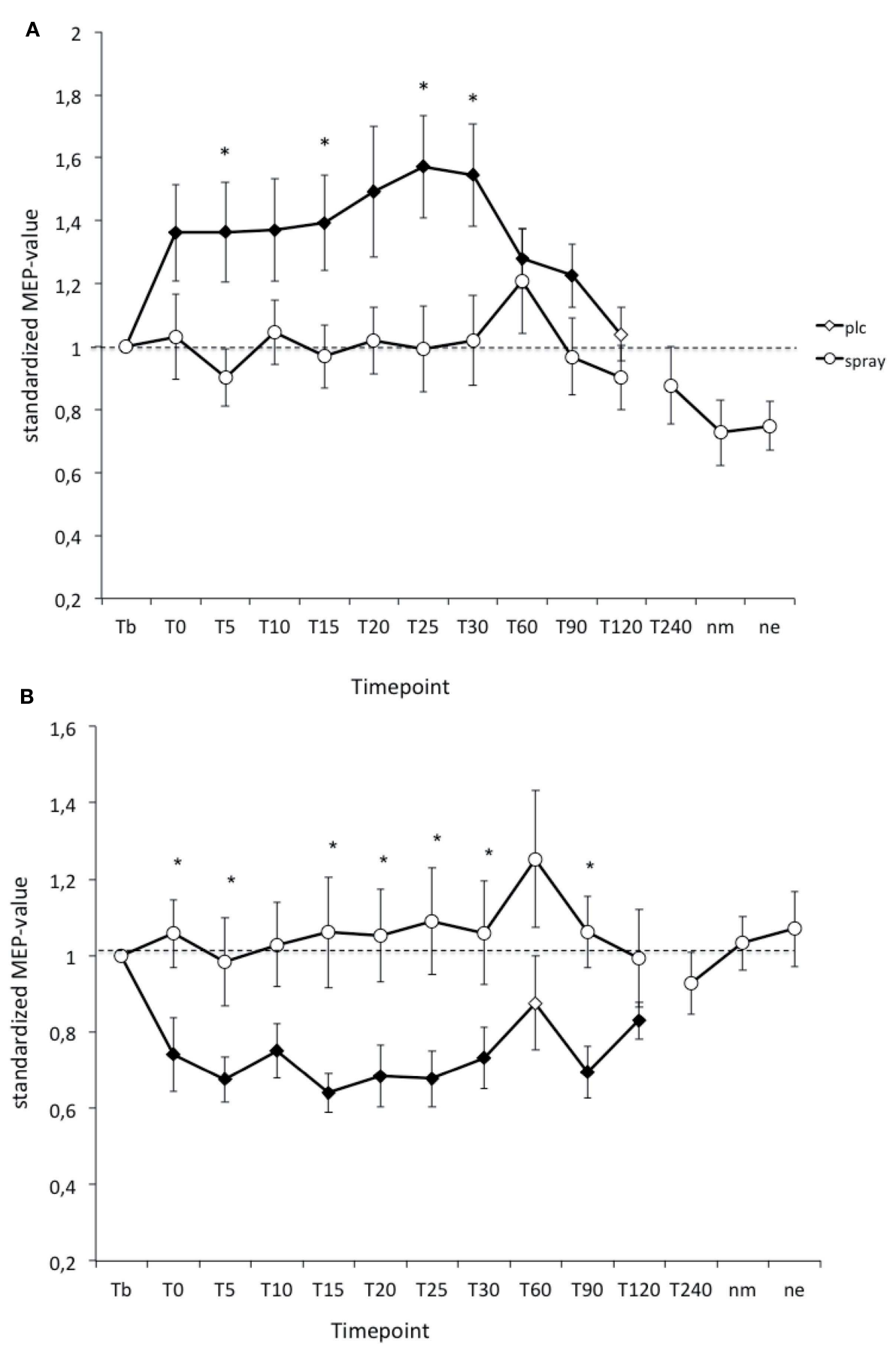

FIGURE 3 | (A,B) Nicotinergic impact on paired associative stimulation (PAS) induced neuroplasticity. Shown are the graphs with motor evoked potentials (MEP) standardized to the baseline on the $Y$-axis plotted against different time points post-stimulation on the $X$-axis. Filled symbols indicate statistically significant deviations from baseline and asterisks indicate significant

differences between the placebo medication and nicotine conditions (Student's $t$-test, paired, two-tailed, $p<0.05)$. nm, Next morning; ne, next evening; plc, placebo. Tb, Baseline MEP-amplitude before begin of the stimulation protocols (standardized). Error bars indicate standard error of mean.

and the homomeric $\alpha 7$ subunit, both exhibiting different pharmacological and physiological properties (Jones et al., 1999), and both increasing intracellular calcium levels by serving as pre- and postsynaptic ligand-gated calcium channels. As the after-effects of tDCS and PAS are likewise calcium-dependent
(Stefan et al., 2002; Nitsche et al., 2003), a possible effect of nicotine on neuroplasticity might be alteration of intracellular calcium levels. The amount of intracellular calcium determines if inhibitory, facilitatory, or no plasticity is induced (Lisman, 2001; Misonou et al., 2004). Given that nicotine enhances intracellular 

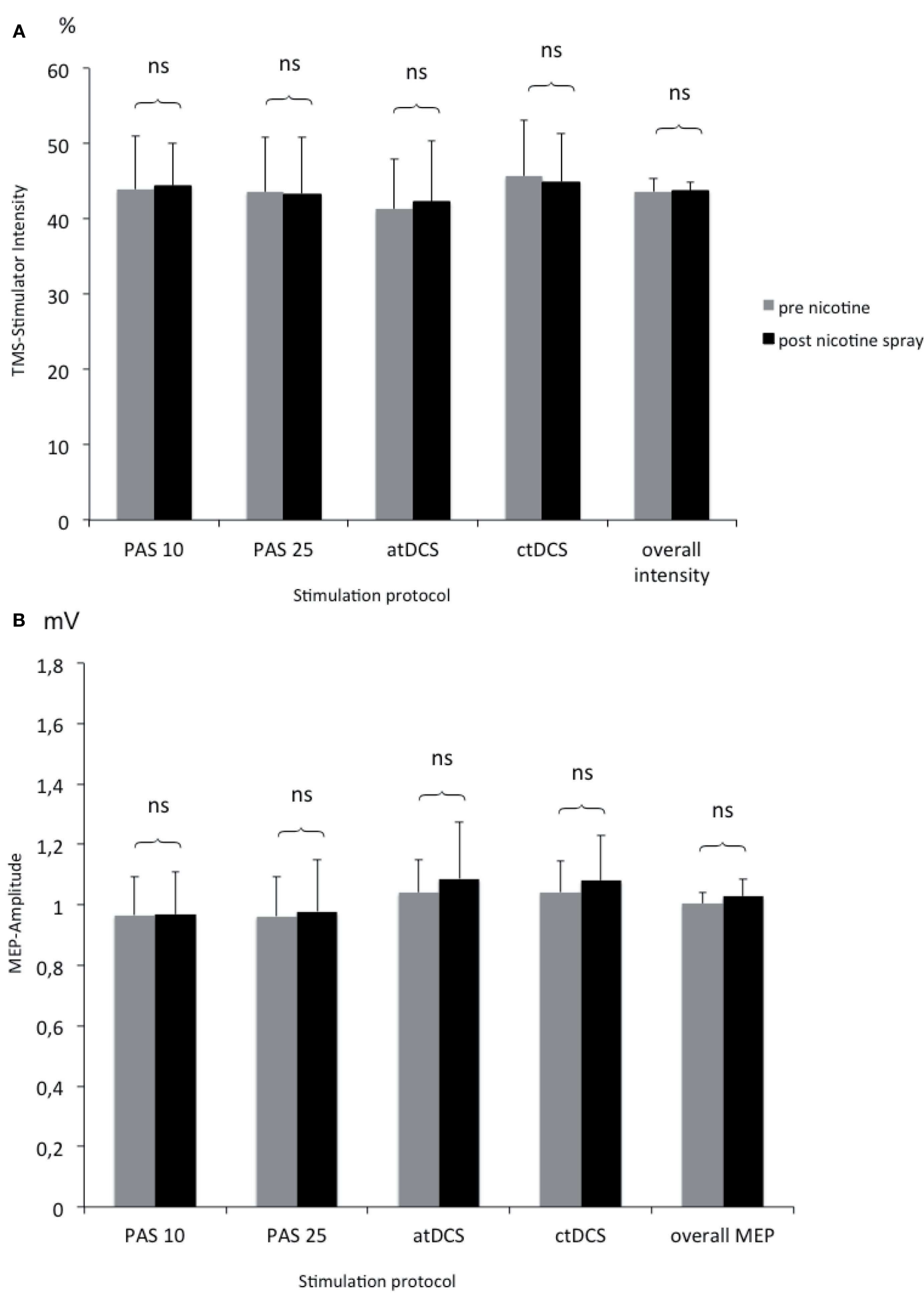

FIGURE 4 | (A,B) Comparison of TMS-stimulator intensity and MEP amplitudes before and after nicotine spray administration. Shown are the TMS-stimulator intensity as percentage of maximum stimulator output (A) and respective motor evoked potentials [MEP amplitudes in $\mathrm{mV}$; (B)] before and after nicotine spray administration for the different interventions (atDCS, ctDCS, PAS-25, PAS-10). ns, Non significant; $\mathrm{mV}$, millivolt. calcium concentration via activation of the respective nicotinic receptors, nicotine administration might have caused a calcium concentration that overshoots the limit for LTP-like induction elicited by atDCS and PAS-25, thus resulting in an extinction of after-effects. The same might be true for LTD-induction processes. 
This proposed mechanism of action is however hypothetical and highly speculative presently and needs to be further explored experimentally in the future. Due to the complex impact of nicotine on other neuromodulators and - transmitters, alternative mechanisms of action cannot be ruled out.

\section{GENERAL REMARKS}

The results of our study demonstrate that nicotine spray influences neuroplasticity in non-smoking humans prominently. Nicotine spray in abolishes/reduces focal and non-focal plasticity. Here the connection to cognitive studies is not easily drawn, since the nicotinic effect on cognition and memory in non-smokers is discussed controversially. A review of Heishman (1998) reported about no true enhancement of sensory ability, selective attention, learning, and other cognitive abilities (e.g., problem solving, reasoning) in non-smoking subjects. Ernst et al. (2001) describe a nicotinic improvement of reaction time, but no effect on working memory in non-smokers. Poltavski and Petros (2005) have even shown a decrement in working memory in non-smokers. To further complicate argumentation the majority of the studies have used nicotine devices other than nicotine spray (nicotine patch, cigarets, inhaler), thus comparability to these studies is difficult because of different bioavailability. Only few studies have used nicotine spray in non-smokers and have found amelioration in rapid visual information processing (Rusted and Alvares, 2008) and fine motor-skills (Perkins et al., 2008). Potential connections of nicotinergic changes in LTP- and LTP-like plasticity and alterations in cognitive functions in smokers and non-smokers have to be explored more directly and intensively in the future. To clarify the specific receptor mechanisms, different nicotinic receptor subtypes have to be examined by pharmacological interventions (agonists and antagonists). Moreover further exploration of genetic differences between smokers and non-smokers might give further insight into the question, why people develop a nicotine addiction.

\section{LIMITING CONDITIONS}

Some limitations of the present study should be taken into account. The study was conducted in a single-blinded manner, so that the person carrying out the experiments knew about the condition of the subject (nicotine spray vs. placebo), thus delivering a possible confounding factor. Furthermore, TMS-measurements of

\section{REFERENCES}

Albuquerque, E. X., Pereira, E. F., Alkondon, M., and Rogers, S. W. (2009). Mammalian nicotinic acetylcholine receptors: from structure to function. Physiol. Rev. 89, 73-120. [Review].

Alkondon, M., Pereira, E. F., Almeida, L. E., Randall, W. R., and Albuquerque, E. X. (2000). Nicotine at concentrations found in cigarette smokers activates and desensitizes nicotinic acetylcholine receptors in CA1 interneurons of rat hippocampus. Neuropharmacology 39, 2726-2739.

Barr, R. S., Pizzagalli, D. A., Culhane, M. A., Goff, D. C., and Evins, A. E. (2008). A single dose of nicotine enhances reward responsiveness in nonsmokers: implications for development of dependence. Biol. Psychiatry 1, 1061-1065.

Burnashev, N. (1998). Calcium permeability of ligand-gated channels. Cell Calcium 24, 325-332.

Dajas-Bailador, F., and Wonnacott, S. (2004). Nicotinic acetylcholine receptors and the regulation of neuronal signalling. Trends Pharmacol. Sci. 25, 317-324. [Review].

Ernst, M., Heishman, S. J., Spurgeon, L., and London, E. D. (2001). Smoking history and nicotine effects on cognitive performance. Neuropsychopharmacology 25, 313-319.

cortical excitability were not performed until the next day in the placebo group, which complicates evaluation of nicotinic longlasting effects on plasticity. Another limitation is the fact, that we cannot exclude non-linear dose-dependent effects of nicotine spray, since we performed the study with a stable dose of $1 \mathrm{mg}$. Dose-related effects on cortical excitability have p.e. been described for dopamine (Monte-Silva et al., 2009) and possible have to be taken into account also for other substances. This might be of special importance for the physiological effects of neuromodulators. Another restriction is that we did not obtain blood levels of nicotine, thus it cannot be excluded completely that different effects of nicotine patch and spray applications on plasticity are at least partially caused by different blood concentrations. It might furthermore complicate the comparability of the results of this study with other studies as well as cognitive testing. Still former pharmacological studies have measured blood levels in subjects taking $1 \mathrm{mg}$ nicotine spray. With an average of $8-9 \mathrm{ng} / \mathrm{ml}$, the blood level did not differ from the blood levels obtained after administration of nicotine patch $15 \mathrm{mg} / 16 \mathrm{~h}(8,9 \mathrm{ng} / \mathrm{ml})$, which is the dosage we had chosen for the patch study (Tønnesen et al., 1991; Pomerleau et al., 1992; Thirugnanasambandam et al., 2011).

Moreover it should be mentioned that the explanation and discussion of the results is only hypothetical presently as currently direct correlation to cognitive studies is still missing. Possible future studies might test this linking between cortical excitability changes and impact on cognitive functions more directly and give further insight in the mechanisms of nicotine in smokers and non-smokers. Since chronic nicotine spray administration has fast and similar effects on craving and withdrawal symptoms than patch (Hajek et al., 1999) it would be further interesting in future possible studies to explore long-term effects of nicotine spray on neuroplasticity.

\section{ACKNOWLEDGMENTS}

The study was supported by the Deutsche Forschungsgemeinschaft (DFG grant NI683/4-1 “Toward risk prediction of nicotine dependency by exploring individual limits if cortical neuroplasticity in humans"; NI 683/4-2 "Impact of the nicotinergic alpha7 receptor in smokers and non-smokers)" within the DFG priority program "Nicotine: molecular and physiological effects in central nervous system."

Flores, C. M., Rogers, S. W., Pabreza, L. A., Wolfe, B. B., and Kellar, K. J. (1992). A subtype of nicotinic cholinergic receptor in rat brain is composed of alpha 4 and beta 2 subunits and is up-regulated by chronic nicotine treatment. Mol. Pharmacol. 41, 31-37.

Hahn, B., and Stolerman, I. P. (2002). Nicotine-induced attentional enhancement in rats: effects of chronic exposure to nicotine. Neuropsychopharmacology 27, 712-722.

Hajek, P., West, R., Foulds, J., Nilsson, F., Burrows, S., and Meadow, A. (1999). Randomized comparative trial of nicotine polacrilex, a transdermal patch, nasal spray, and an inhaler. Arch. Intern. Med. 159, 2033-2038.

Heishman, S. J. (1998). What aspects of human performance are truly enhanced by nicotine? Addiction 93, 317-320. [Review].

Huerta, P. T., and Lisman, J. E. (1995). Bidirectional synaptic plasticity induced by a single burst during cholinergic theta oscillation in CA1 in vitro. Neuron 15, 1053-1063. Jones, H. E., Garrett, B. E., and Griffiths, R. R. (1999). Subjective and physiological effects of intravenous nicotine and cocaine in cigarette smoking cocaine abusers. J. Pharmacol. Exp. Ther. 288, 188-197. 
Kleykamp, B. A., Jennings, J. M., Blank, M. D., and Eissenberg, T. (2005). The effects of nicotine on attention and working memory in neversmokers. Psychol. Addict. Behav. 19, 433-438.

Kumari, V., Gray, J. A., ffytche, D. H., Mitterschiffthaler, M. T., Das, M., Zachariah, E., et al. (2003). Cognitive effects of nicotine in humans: an fMRI study. Neuroimage 19, 1002-1013.

Kuo, M. F., Grosch, J., Fregni, F., Paulus, W., and Nitsche, M. A. (2007). Focusing effect of acetylcholine on neuroplasticity in the human motor cortex. J. Neurosci. 27, 14442-14447.

Levin, E. D., Briggs, S. J., Christopher, N. C., and Auman, J. T. (1994). Working memory performance and cholinergic effects in the ventral tegmental area and substantia nigra. Brain Res. 657, 165-170.

Levin, E. D., McClernon, F. J., and Rezvani, A. H. (2006). Nicotinic effects on cognitive function: behavioral characterization, pharmacological specification, and anatomic localization. Psychopharmacology (Berl.) 184, 523-539. [Review].

Lisman, J. E. (2001). Three $\mathrm{Ca}^{2+}$ levels affect plasticity differently: the LTP zone, the LTD zone and no man's land. J. Physiol. (Lond.) 532(Pt 2), 285.

Mansvelder, H. D., and McGehee, D. S. (2000). Long-term potentiation of excitatory inputs to brain reward areas by nicotine. Neuron 27 , 349-357.

Matsuyama, S., Matsumoto, A., Enomoto, T., and Nishizaki, T. (2000). Activation of nicotinic acetylcholine receptors induces long-term potentiation in vivo in the intact mouse dentate gyrus. Eur. J. Neurosci. 12, 3741-3747.

Misonou, H., Mohapatra, D. P., Park, E. W., Leung, V., Zhen, D., and Misonou, K., et al. (2004). Regulation of ion channel localization and phosphorylation by neuronal activity. Nat. Neurosci. 7, 711-718.

Monte-Silva, K., Kuo, M. F., Thirugnanasambandam, N., Liebetanz, D., Paulus, W., and Nitsche, M. A. (2009). Dose-dependent inverted Ushaped effect of dopamine (D2like) receptor activation on focal and nonfocal plasticity in humans. J. Neurosci. 13, 6124-6131.

Mukhin, A. G., Kimes, A. S., Chefer, S. I., Matochik, J. A., Contoreggi, C. S., Horti, A. G., et al. (2008). Greater nicotinic acetylcholine receptor density in smokers than in nonsmokers: a PET study with 2-18F-FA-85380. J. Nucl. Med. 49, 1628-1635.

Nitsche, M. A., Fricke, K., Henschke, U., Schlitterlau, A., Liebetanz, D., Lang, N., et al. (2003). Pharmacological modulation of cortical excitability shifts induced by transcranial direct current stimulation in humans. J. Physiol. (Lond.) 15(Pt 1), 293-301.

Nitsche, M. A., Jaussi, W., Liebetanz, D., Lang, N., Tergau, F., and Paulus, W. (2004). Consolidation of human motor cortical neuroplasticity by Dcycloserine. Neuropsychopharmacology 29, 1573-1578.

Nitsche, M. A., and Paulus, W. (2000). Excitability changes induced in the human motor cortex by weak transcranial direct current stimulation. J. Physiol. (Lond.) 527, 633-639.

Nitsche, M. A., and Paulus, W. (2001). Sustained excitability elevations induced by transcranial DC motor cortex stimulation in humans. Neurology 57, 1899-1901.

Nitsche, M. A., Roth, A., Kuo, M. F., Fischer, A. K., Liebetanz, D., and Lang, N., et al. (2007). Timing-dependent modulation of associative plasticity by general network excitability in the human motor cortex. J. Neurosci. 27, 3807-3812.

Perkins, K. A., Lerman, C., Coddington, S. B., Jetton, C., Karelitz, J. L., Scott, J. A., et al. (2008). Initial nicotine sensitivity in humans as a function of impulsivity. Psychopharmacology (Berl.) 200, 529-544.

Poltavski, D. V., and Petros, T. (2005). Effects of transdermal nicotine on prose memory and attention in smokers and nonsmokers. Physiol Behav. 17, 833-843.

Pomerleau, O. F., Flessland, K. A., Pomerleau, C. S., and Hariharan, M. (1992). Controlled dosing of nicotine via an Intranasal Nicotine Aerosol Delivery Device (INADD). Psychopharmacology (Berl.) 108, 519-526.

Purpura, D. P., and McMurtry, J. G. (1965). Intracellular activities and evoked potential changes during polarization of motor cortex. J. Neurophysiol. 28, 166-185.

Rioult-Pedotti, M. S., Friedman, D., and Donoghue, J. P. (2000). Learninginduced LTP in neocortex. Science 290, 533-536.

Rioult-Pedotti, M. S., Friedman, D., Hess, G., and Donoghue, J. P. (1998). Strengthening of horizontal cortical connections following skill learning. Nat. Neurosci. 1, 230-234.

Rusted, J. M., and Alvares, T. (2008). Nicotine effects on retrieval-induced forgetting are not attributable to changes in arousal. Psychopharmacology (Berl.) 196, 83-92.

Sawada, S., Yamamoto, C., and OhnoShosaku, T. (1994). Long-term potentiation and depression in the dentate gyrus, and effects of nicotine. Neurosci. Res. 20, 323-329.

Schneider, N. G., Olmstead, R., Mody, F. V., Doan, K., Franzon, M., Jarvik, M. E., et al. (1995). Efficacy of a nicotine nasal spray in smoking cessation: a placebo-controlled, double-blind trial. Addiction 90, 1671-1682.

Stefan, K., Kunesch, E., Benecke, R. Cohen, L. G., and Classen, J. (2002). Mechanisms of enhancement of human motor cortex excitability induced by interventional paired associative stimulation. J. Physiol. (Lond.) 543(Pt 2), 699-708.

Stefan, K., Kunesch, E., Cohen, L G., Benecke, R., and Classen, J. (2000). Induction of plasticity in the human motor cortex by paired associative stimulation. Brain 123 572-584.

Sutherland, G., Russell, M. A., Stapleton, J., Feyerabend, C., and Ferno, O. (1992). Nasal nicotine spray: efficacy of a nicotine nasal spray in smoking cessation: a placebo-controlled, double-blind trial. Addiction 90, 1671-1682.

Swayne, O. B., Teo, J. T., Greenwood, R. J., and Rothwell, J. C. (2009) The facilitatory effects of intermittent theta burst stimulation on corticospinal excitability are enhanced by nicotine. Clin. Neurophysiol. 120, 1610-1615.

Thiel, C. M., Zilles, K., and Fink, G. R. (2005). Nicotine modulates reorienting of visuospatial attention and neural activity in human parietal cortex. Neuropsychopharmacology 30, 810-820.

Thirugnanasambandam, N., Grundey, J., Adam, K., Drees, A., and Skwirba, A. C. (2011). Nicotinergic impact on focal and non-focal neuroplasticity induced by non-invasive brain stimulation in non-smoking humans. Neuropsychopharmacology 36, 879-886.

Tønnesen, P., Nørregaard, J., Simonsen, K., and Säwe, U. (1991). A doubleblind trial of a 16-h transdermal nicotine patch in smoking cessation. N. Engl. J. Med. 325, 311-315

Weise, D., Schramm, A., Stefan, K., Wolters, A., Reiners, K., Naumann, M., et al. (2006). The two sides of associative plasticity in writer's cramp. Brain 129(Pt 10), 2709-2721.

Wolters, A., Sandbrink, F., Schlottmann, A., Kunesch, E., Stefan, K., Cohen, L. G., et al. (2003). A temporally asymmetric Hebbian rule governing plasticity in the human motor cortex. $J$. Neurophysiol. 89, 2339-2345.

Conflict of Interest Statement: The authors declare that the research was conducted in the absence of any commercial or financial relationships that could be construed as a potential conflict of interest.

Received: 06 May 2012; accepted: 05 October 2012; published online: 26 October 2012.

Citation: Grundey J, Thirugnanasambandam $N$, Kaminsky $K$, Drees $A$, Skwirba AC, Lang N, Paulus $W$ and Nitsche MA (2012) Rapid effect of nicotine intake on neuroplasticity in non-smoking humans. Front. Pharmacol. 3:186. doi: 10.3389/fphar.2012.00186 This article was submitted to Frontiers in Neuropharmacology, a specialty of Frontiers in Pharmacology. Copyright (c) 2012 Grundey, Thirugnanasambandam, Kaminsky, Drees, Skwirba, Lang, Paulus and Nitsche. This is an open-access article distributed under the terms of the Creative Commons Attribution License, which permits use, distribution and reproduction in other forums, provided the original authors and source are credited and subject to any copyright notices concerning any third-party graphics etc. 